\title{
Color breaking baryogenesis
}

\author{
Michael J. Ramsey-Musolf, ${ }^{1,2, *}$ Graham White, ${ }^{3,4, \dagger}$ and Peter Winslow ${ }^{1, \$}$ \\ ${ }^{1}$ Physics Department, University of Massachusetts Amherst, Amherst, Massachusetts 01003, USA \\ ${ }^{2}$ Kellogg Radiation Laboratory, California Institute of Technology, Pasadena, California 91125, USA \\ ${ }^{3}$ ARC Centre of Excellence for Particle Physics, Monash University, Victoria 3800, Australia \\ ${ }^{4}$ TRIUMF, 4004 Wesbrook Mall, Vancouver, British Columbia V6T 2A3, Canada
}

(Received 11 September 2017; published 8 June 2018)

\begin{abstract}
We propose a scenario that generates the observed baryon asymmetry of the Universe through a multistep phase transition in which SU(3) color symmetry is first broken and then restored. A spontaneous violation of $B-L$ conservation leads to a contribution to the baryon asymmetry that becomes negligible in the final phase. The baryon asymmetry is therefore produced exclusively through the electroweak mechanism in the intermediate phase. We illustrate this scenario with a simple model that reproduces the observed baryon asymmetry. We discuss how future electric dipole moment and collider searches may probe this scenario, though future electric dipole moment searches would require an improved sensitivity of several orders of magnitude.
\end{abstract}

DOI: 10.1103/PhysRevD.97.123509

\section{INTRODUCTION}

The origin of the cosmic matter-antimatter asymmetry remains one of the outstanding open questions at the interface of cosmology with particle and nuclear physics. The Planck experiment determines that the baryon asymmetry of the Universe (BAU) is [1]

$$
\frac{n_{B}}{s} \equiv Y_{B}=(8.59 \pm 0.11) \times 10^{-11}
$$

where $n_{B}(s)$ is the baryon number (entropy) density. To dynamically generate the BAU one must fulfil three Sakharov conditions [2]: baryon number violation, C and $C P$ violation, and a departure from equilibrium. The Standard Model (SM) cannot explain the matter-antimatter asymmetry as it fails to provide sufficient $C P$ violation [3-5] and the required out-of-equilibrium conditions [6-9]. As such, many beyond SM scenarios have arisen to accommodate this need.

For many years, electroweak baryogenesis (EWBG) has been one of the most attractive scenarios for explaining the BAU [10-12]. The main reason for this interest has been its testable nature due to its strong connection with

\footnotetext{
*mjrm@physics.umass.edu †graham.white@monash.edu "pwinslow@physics.umass.edu
}

Published by the American Physical Society under the terms of the Creative Commons Attribution 4.0 International license. Further distribution of this work must maintain attribution to the author(s) and the published article's title, journal citation, and DOI. Funded by SCOAP ${ }^{3}$. the weak scale. However, successful electroweak baryogenesis requires new bosonic states with masses near the weak scale and significant couplings to the Higgs boson in order to generate a strongly first-order electroweak phase transition (EWPT). One of the most widely considered possibilities, the minimal supersymmetric standard model (MSSM) with relatively light top squarks, appears to be in considerable tension with LHC data, e.g., see [13,14] (however, see also [15]). In this context, it is worth asking if there are well-motivated and testable modifications to the EWBG paradigm.

The rich landscape of phase structures in condensed matter systems suggests that the thermal history of symmetries in the Universe might be more exotic than the conventional scenario involving a single instance of electroweak symmetry breaking at a temperature $T_{\mathrm{EW}} \sim 100 \mathrm{GeV}$. This possibility has been suggested in Weinberg's analysis of gauge symmetries at finite temperature [16], and subsequently followed up by several authors [17-26]. As observed in Ref. [16], for example, Rochelle salt has the remarkable property of first undergoing a symmetry-breaking transition as the temperature is lowered, followed by a symmetry-restoring transition at lower temperature [27]. This raises the fascinating possibility that a similar phenomenon may occur in gauge theories [16].

In light of this possibility and the constraints on the EWBG paradigm, we consider a multistep phase transition beginning with a symmetric Universe at high temperature, followed by the spontaneous breaking of $\mathrm{SU}(3)_{C}$ as the Universe cools and ending with its subsequent restoration. Although there have been studies of multistep phase 
transitions incorporating $\mathrm{SU}(3)_{C}$-breaking [7,23,25], only the last has given a viable mechanism to break $\mathrm{SU}(3)_{C}$ symmetry and restore it at zero temperature. ${ }^{1}$

In this study, we follow the general setup of Ref. [25] where $\mathrm{SU}(3)_{C}$-breaking is induced by colored scalars obtaining a vacuum expectation value (vev) during the first transition, which breaks both the color $\mathrm{SU}(3)_{C}$ and electroweak (EW) $\mathrm{SU}(2)_{L} \times \mathrm{U}(1)_{Y}$ symmetries of the $\mathrm{SM}$. This vev is then erased during the subsequent transition to the present "Higgs phase," wherein only the neutral component of the Higgs doublet obtains a vev. We refer to these two transitions as the color-breaking $(\mathrm{CoB})$ and $\mathrm{EW}$ phase transitions, respectively (though technically both break EW symmetry). We develop a full working scenario of baryogenesis under these conditions, which we refer to as color-breaking baryogenesis (CoBBG). We will focus our attention on the $C P$ violation and charge transport dynamics and not the dynamics of the phase transition that was previously studied in Ref. [25].

To demonstrate this new paradigm, we introduce two new scalar fields, $\mathrm{C}_{1,2}$, that are charged under $\mathrm{SU}(3)_{C}$ as well as $\mathrm{SU}(2)_{L} \times U(1)_{Y}$. In order to prevent the existence of stable colored relics, we take these fields to interact with the standard model as leptoquarks through Yukawa-type interactions. With this field content, the thermal history of symmetry breaking is

$$
\begin{aligned}
& S U(3)_{C} \times S U(2)_{L} \times U(1)_{Y} \\
\stackrel{T_{1}}{\rightarrow} & S U(2)_{C} \times U(1)_{X_{1}} \times U(1)_{X_{2}} \\
\stackrel{T_{2}}{\rightarrow} & S U(3)_{C} \times U(1)_{\mathrm{EM}},
\end{aligned}
$$

where $X_{1,2}$ denote two independently conserved U(1) charges during the $\mathrm{CoB}$ phase that accompany a residual color $\mathrm{SU}(2)_{C}$ symmetry. The BAU is generated during the first phase transition at temperature $T_{1}$, with the Sakharov conditions realized as follows:

(1) Baryon number conservation is violated in two ways: the usual electroweak sphalerons anomalously violating $B+L$ and spontaneous violation of $B-L$ in the color-breaking phase, since the leptoquark fields $C_{j}$ carry $B-L$.

(2) The leptoquark-quark-lepton Yukawa couplings contain new $C P$-violating complex phases that source the generation of charge asymmetries during the first transition.

(3) The spontaneous breaking of $\mathrm{SU}(3)_{C}$ symmetry proceeds via a strongly first order phase transition, resulting in nucleation of $\mathrm{CoB}$ bubbles and, thereby, satisfying the out-of-equilibrium requirement.

\footnotetext{
${ }^{1}$ We note that Ref. [25] did not analyze the strength of the phase transition or which parts of the parameter space have sufficiently fast tunneling. A detailed investigation into color breaking phase transitions is the subject of ongoing research.
}

During the second transition at temperature $T_{2}$, the BAU produced during the first step inside the $\mathrm{CoB}$ phase is transferred to the Higgs phase. So long as the second transition does not permit re-excitation of the unbroken phase EW sphalerons or injection of significant entropy, the first phase BAU will not be washed out or diluted when the second transition occurs.

During the first step, the BAU produced via electroweak sphalerons is directly analogous to EWBG. Electroweak sphalerons are unsuppressed in the symmetric phase. $C P$ violating interactions with the walls of the expanding $\mathrm{CoB}$ phase bubbles creates a total left handed number density that biases the sphalerons at the bubble exteriors. This produces a net $B+L$ asymmetry, some of which is swept up by the advancing bubble wall. For a sufficiently strong first-order $\mathrm{CoB}$ transition, the broken phase $\mathrm{EW}$ sphaleron transitions will be sufficiently quenched by the $C_{j}$ vevs so as to preclude washout of the $B+L$ asymmetry.

The second mechanism for violating baryon number conservation involves the spontaneous violation of $B-L$ number conservation by the $C_{j}$ vevs. The total $B-L$ inside and outside the bubble is zero; however, a nonzero $B-L$ density is trapped inside the expanding bubble. The size of this contribution, however, is negligible as the $B-L$ density relaxes to zero within a trillionth of the Hubble length at the time of nucleation and will continue to diffuse. On the other hand, the $B+L$ asymmetry is effectively conserved deep within the color-broken phase and persists into the electroweak phase. The net BAU is, thus, dominated by the conventional $B+L$ generating EWBG mechanism. We find that given the present phenomenological constraints from collider searches and electric dipole moments, the resulting BAU can be comparable in magnitude to the observed asymmetry.

We organize our discussion of this scenario as follows. In Sec. II we define our exact choice of model to illustrate this scenario and Sec. III elaborates on the symmetrybreaking patterns associated with the multistep phase transition. In Sec. IV we analyze all issues of charge transport including local equilibrium considerations, derivation of quantum transport equations, and our results including the contribution from the electroweak mechanism. Section V discusses the zero temperature phenomenology before we conclude in Sec. VI.

\section{THE MODEL}

Our illustrative model consists of the SM plus two scalar leptoquark fields, $C_{1,2}$, that must be charged under $\mathrm{SU}(3)_{C}$ and $\mathrm{SU}(2)_{L}$ in order to catalyze a $\mathrm{CoB}$ phase transition and quench electroweak sphalerons during this transition. In general, there are three scalar leptoquark representations that couple to $\mathrm{SM}$ fermions and have nontrivial $\mathrm{SU}(2)_{L}$ quantum numbers [28], $(3,3)_{-1 / 3},(3,2)_{7 / 6}$, and $(3,2)_{1 / 6}$. We seek a model that has the minimum number of free 
parameters, is least constrained phenomenologically, and does not enable any baryon number-violating processes at zero temperature.

The $(3,3)_{-1 / 3}$ representation does not pass these requirements as it couples to both $Q_{L} Q_{L}$ and $Q_{L}^{\dagger} L_{L}^{\dagger}$, violating baryon number explicitly at tree level. The $(3,2)_{7 / 6}$ representation admits an enhanced $\mu \rightarrow e \gamma$ rate by virtue of it coupling to both $Q_{L}^{\dagger} e_{R}$ and $u_{R}^{\dagger} L_{L}$. The same enhancement also appears in 1-loop logarithmically divergent contributions to the charged lepton mass matrix, leading to nontrivial naturalness constraints even if the leptoquarks only couple to third generation particles [29]. In contrast, the $(3,2)_{1 / 6}$ representation only couples to $d_{R}^{\dagger} L_{L}$, so it is not subject to the above phenomenological constraints. Furthermore, it has no perturbative baryon number violation and can catalyze gauge-coupling unification [30]. We therefore use this representation to illustrate the mechanism of CoBBG.

Given the quantum numbers of the $C_{1,2}$ fields, we denote them as

$$
C_{j \alpha}=\left(\begin{array}{c}
\chi_{j \alpha}^{2 / 3} \\
\chi_{j \alpha}^{-1 / 3}
\end{array}\right) \quad j=1,2
$$

where Roman and Greek subscripts indicate the field and $\mathrm{SU}(3)_{C}$ indices, respectively, while superscripts represent the electromagnetic charges of the $\mathrm{SU}(2)_{L}$ component fields. The most general Yukawa interaction for this representation is

$$
\mathcal{L}_{Y} \supset \bar{d}_{R}^{\alpha}\left(Y^{1} C_{1 \alpha}+Y^{2} C_{2 \alpha}\right) L+\text { H.c. }
$$

where flavor indices have been suppressed and the $\mathrm{SU}(2)_{L}$ contraction is

$$
C_{j \alpha} L \equiv \chi_{j \alpha}^{2 / 3} e_{L}-\chi_{j \alpha}^{-1 / 3} \nu_{L}
$$

The $3 \times 3$ Yukawa matrices, $Y^{1}$ and $Y^{2}$, couple righthanded down-type quarks $\left(d_{R}, s_{R}, b_{R}\right)$ to left-handed leptons $\left(e_{L}, \mu_{L}, \tau_{L}\right)$ and neutrinos $\left(\nu_{e L}, \nu_{\mu L}, \nu_{\tau L}\right)$. These new Yukawa matrices are arbitrary. However, the absence of large flavor-changing neutral currents (FCNC) combined with LHC constraints being significantly more stringent for leptoquarks coupled to first- or second-generation fermions [31] suggests a hierarchical structure where $\bar{b}_{R}-\nu_{\tau L}$ couplings are dominant. Consequently, we take

$$
Y^{i}=\operatorname{diag}\left(0,0, \tilde{y}_{i}\right),
$$

where the zeros here indicate subleading couplings that we neglect in our analysis. Consequently, there is only one rephasing invariant $C P$ phase, and it is the relative phase
$\operatorname{Im}\left(\tilde{y}_{1}^{*} \tilde{y}_{2}\right)$. This is the minimum structure necessary to illustrate the CoBBG mechanism.

Note that in Ref. [25] it was found that in order to have a phenomenologically viable scenario where color symmetry is broken and restored, one requires there to be gauge singlets in the model. The gauge singlet allows the leptoquark mass to be a $\mathrm{TeV}$ or higher and can result in the leptoquark mass during the color-breaking phase to substantially differ from its zero temperature value in the standard model phase. In this paper we ignore gauge singlets and leave such features to future work.

\section{SPONTANEOUS SYMMETRY BREAKING OF SU(3) $C$ S SU(2) $)_{L} \times \mathbf{U}(1)_{Y}$}

In this section we describe the pattern of spontaneous symmetry breaking during the multistep phase transition. It is necessary to understand this pattern as the presence of conserved gauge symmetries during the $\mathrm{CoB}$ phase will be used to make significant simplifcations in the next section.

Without loss of generality, we choose the orientation of the color-breaking vevs such that the shifted $C_{j}$ fields are

$$
\left(\begin{array}{c}
\chi_{j \alpha}^{2 / 3} \\
\chi_{j \alpha}^{-1 / 3}
\end{array}\right) \rightarrow\left(\begin{array}{c}
\chi_{j \alpha}^{2 / 3} \\
\varphi_{j} \delta_{\alpha}^{3}+\sigma_{j \alpha}^{-1 / 3}
\end{array}\right) \quad j=1,2
$$

where $\varphi_{j}$ are the vevs and the $\delta_{\alpha}^{3}$ singles out a direction in $\mathrm{SU}(3)_{C}$ space. In order to identify the symmetry-breaking pattern, we examine the gauge boson mass spectrum in the CoB phase.

Neglecting fluctuations around the vevs, the gauge boson mass spectrum is given by

$$
\begin{aligned}
& \sum_{j}\left|D_{\mu}\left\langle C_{j \alpha}\right\rangle\right|^{2} \\
& =\varphi_{C B}^{2}\left[e_{S}^{2}\left(G_{\mu}^{+, 45} G^{-, 45 \mu}+G_{\mu}^{+, 67} G^{-, 67 \mu}\right)+e_{W}^{2} W_{\mu}^{+} W^{-\mu}\right. \\
& \left.+\left(\begin{array}{lll}
W_{\mu}^{3} & B_{\mu} & G_{\mu}^{8}
\end{array}\right) \frac{\mathcal{M}^{2}}{2}\left(\begin{array}{c}
W^{3 \mu} \\
B^{\mu} \\
G^{8 \mu}
\end{array}\right)\right]
\end{aligned}
$$

where $\varphi_{C B}^{2} \equiv \varphi_{1}^{2}+\varphi_{2}^{2}$ and the hypercharge, weak, and strong gauge couplings have been normalized as

$$
\left(e_{Y}, e_{W}, e_{S}\right) \equiv\left(g_{Y} / \sqrt{2}, g_{W} / \sqrt{2}, g_{S} / \sqrt{2}\right) .
$$

The $G_{\mu}^{ \pm, i j} \equiv \frac{1}{\sqrt{2}}\left(G_{\mu}^{i} \mp i G_{\mu}^{j}\right)$ fields correspond to the well-known $\mathrm{SU}(3)_{C}$ generators of isospin $(i j=12)$, $\mathrm{U}$ spin $(i j=45)$, and $\mathrm{V}$ spin $(i j=67)$, while the $W^{ \pm} \equiv \frac{1}{\sqrt{2}}\left(W_{\mu}^{1} \mp i W_{\mu}^{2}\right)$ fields correspond to the familiar generators of weak isospin. 


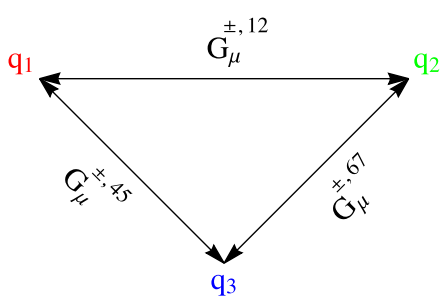

FIG. 1. The interaction pattern of gauge fields corresponding to $\mathrm{SU}(3)_{C}$ generators of isospin $\left(G_{\mu}^{ \pm, 12}\right), \mathrm{U}$ spin $\left(G_{\mu}^{ \pm, 45}\right)$, and $\mathrm{V}$ spin $\left(G_{\mu}^{ \pm, 67}\right)$ and fields in the fundamental triplet representation.

The $3 \times 3$ mass matrix takes the form

$$
\mathcal{M}^{2}=\left(\begin{array}{ccc}
e_{W}^{2} & -\frac{e_{W} e_{Y}}{3} & \frac{2 e_{W} e_{S}}{\sqrt{3}} \\
-\frac{e_{W} e_{Y}}{3} & \frac{e_{Y}^{2}}{9} & -\frac{2}{3 \sqrt{3}} e_{S} e_{Y} \\
\frac{2 e_{W} e_{S}}{\sqrt{3}} & -\frac{2}{3 \sqrt{3}} e_{S} e_{Y} & \frac{4}{3} e_{S}^{2}
\end{array}\right) \text {. }
$$

This matrix has only one nonzero eigenvalue, implying the presence of two unbroken and one broken U(1) gauge symmetries present in the $\mathrm{CoB}$ phase. We denote the corresponding three mass eigenstate fields as

$$
\left(X_{1 \mu}, X_{2 \mu}, X_{3 \mu}\right)^{T}=\mathcal{U}\left(W_{\mu}^{3}, B_{\mu}, G_{\mu}^{8}\right)^{T}
$$

where $\mathcal{U}$ diagonalizes $\mathcal{M}^{2}$. While $X_{1 \mu}$ and $X_{2 \mu}$ remain massless, mediating long-range forces associated with the unbroken symmetries $\mathrm{U}(1)_{X_{1}}$ and $\mathrm{U}(1)_{X_{2}}, X_{3 \mu}$ develops a mass

$$
m_{X_{3}}^{2}=\varphi_{C B}^{2} / 9\left(12 e_{S}^{2}+9 e_{W}^{2}+e_{Y}^{2}\right)
$$

and thus mediates a short-range force associated with the broken $\mathrm{U}(1)_{X_{3}}$ symmetry. The corresponding charge generators of these $\mathrm{U}(1)$ symmetries are given by

$$
\begin{aligned}
& Q_{X_{1}}=T^{8}-\frac{2}{\sqrt{3}} \tau^{3} \\
& Q_{X_{2}}=\tau^{3}+3 Y \\
& Q_{X_{3}}=T^{8}+\frac{\sqrt{3}}{2} \frac{e_{W}^{2}}{e_{S}^{2}} \tau^{3}-\frac{1}{2 \sqrt{3}} \frac{e_{Y}^{2}}{e_{S}^{2}} Y .
\end{aligned}
$$

The charges $Q_{X_{1}}$ and $Q_{X_{2}}$ are conserved in both the symmetric and color-broken phase. The gauge fields corresponding to the $\mathrm{SU}(3)_{C}$ isospin generators are missing from Eq. (8) and thus remain massless, indicating the existence of an unbroken $\mathrm{SU}(2)_{C}$ subgroup of $\mathrm{SU}(3)_{C}$ in the $\mathrm{CoB}$ phase. This situation effectively distinguishes color state $q_{3}$ from $q_{1}, q_{2}$, indicating that its dynamics should be treated separately in the $\mathrm{CoB}$ phase. This situation is represented graphically in Fig. 1 and further clarified in Sec. IVA. In Sec. IV B we will study the charge transport dynamics of each independent color separately.

\section{BARYOGENESIS}

The BAU calculation is performed in two steps. First, we analyze charge transport dynamics of the relevant number densities in order to calculate the space-time varying $B-L$ and chiral charge densities generated during the strongly first-order $\mathrm{CoB}$ phase transition. Second, we calculate the total left-handed number density that biases the sphalerons resulting in a $B+L$ asymmetry via the EWBG mechanism. Combining the results of contributions yields the net BAU in the $\mathrm{CoB}$ phase.

The dynamics of particle number densities during a first-order phase transition is a highly non-Markovian process that depends on the entire history of the system. In particular, "memory effects" can lead to a resonant boost of both $C P$-violating sources and $C P$-conserving relaxation terms that result from interactions with the spacetime varying vacuum [32-35]. Recall that our model contains a new, $T=0$ rephasing invariant within the leptoquark interaction that results in a new $C P$-violating $(\mathrm{CPV})$ and $(B-L)$-violating $(\mathrm{BLV})$ source $S_{i}^{(\mathrm{CPV}, \mathrm{BLV})}$ for the transport equations

$$
\partial_{\mu} j_{i}^{\mu}=-\sum_{j} \Gamma_{i j} \mu_{j}+S_{i}^{(\mathrm{CPV}, \mathrm{BLV})}
$$

where $j_{i}^{\mu}$ and $\mu_{i}$ are the charge current density and chemical potential, respectively, of particle species $i$, and $\Gamma_{i j}$ are the rates of interactions between species $i$ and $j$.

The computation of the $S_{i}^{(\mathrm{CPV}, \mathrm{BLV})}$ is, in general, quite subtle, and there remain a number of open theoretical issues for the CPV sources involving fermions (for a discussion, see, e.g., Ref. [11] and references therein). The general framework we adopt is the Schwinger-Keldysh closed time path formalism [36-41]. We will work with the vev insertion approximation (VIA), wherein we treat spacetime varying vevs appearing in the $b-\nu$ mass matrix perturbatively to lowest nontrivial order. The diagrammatic representation of $S_{i}^{(\mathrm{CPV}, \mathrm{BLV})}$ in the VIA is shown Fig. 2. We expect that the VIA gives a reasonable guide to the magnitude of the CPV effects and allows one to see structure of the dynamics in our scenario. A more refined treatment including full accounting for flavor oscillations and vev-resummations is in progress $[33,34,42,43]$, and it remains unclear as to whether the VIA yields an overestimate or underestimate. Consequently, we will take our results as indicative of the magnitude of the BAU in our set up and not as numerically definitive.

With these caveats in mind, we apply the techniques in Refs. [35,44], and we obtain 


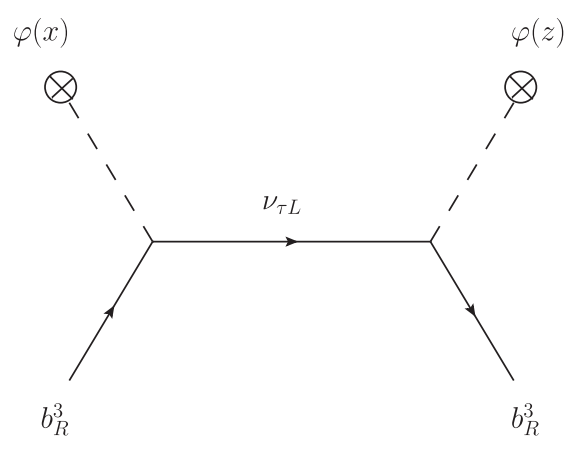

FIG. 2. Interaction between the left-handed tau neutrino and the right-handed 3rd color bottom quark with space-time varying vacuum. This interaction is responsible for new $C P$-violating source.

$$
\begin{aligned}
S_{b}^{(\mathrm{CPV}, \mathrm{BLV})}= & -S_{\nu_{L}}^{(\mathrm{CPV}, \mathrm{BLV})} \\
= & \frac{\operatorname{Im}\left[\tilde{y}_{1} \tilde{y}_{2}\right]}{\pi^{2}} v_{C B}^{2}(z) \frac{\partial \zeta(z)}{\partial t} \int_{0}^{\infty} \frac{k^{2} d k}{\omega_{\nu_{L}} \omega_{b}} \\
& \times \operatorname{Im}\left[\left(\mathcal{E}_{\nu_{L}} \mathcal{E}_{b}+k^{2}\right)\left(\frac{n_{f}\left(\mathcal{E}_{\nu_{L}}\right)+n_{F}\left(\mathcal{E}_{b}\right)}{\left(\mathcal{E}_{\nu_{L}}+\mathcal{E}_{b}\right)^{2}}\right)\right. \\
& \left.+\left(\mathcal{E}_{\nu_{L}} \mathcal{E}_{b}^{*}-k^{2}\right)\left(\frac{n_{f}\left(\mathcal{E}_{\nu_{L}}\right)-n_{F}\left(\mathcal{E}_{b}^{*}\right)}{\left(\mathcal{E}_{b}^{*}-\mathcal{E}_{\nu_{L}}\right)^{2}}\right)\right] .
\end{aligned}
$$

Here, $\tan \zeta(z)$ is the ratio of the vevs of the colored scalars, $\varphi_{2}(z) / \varphi_{1}(z), n_{F}$ is the Fermi-Dirac distribution function, $\omega_{i}=\sqrt{k^{2}+m_{i}^{2}}$, and $\mathcal{E}_{i} \equiv \sqrt{k^{2}+m_{i}^{2}}-i \Gamma_{i}$ with $m_{i}$ and $\Gamma_{i}$ representing the fully corrected thermal mass and width of state $i{ }^{2}$

Denoting the chemical potentials of the left-handed tau neutrino and the third color component of the right-handed bottom quark as $\mu_{\nu_{\tau L}}$ and $\mu_{b_{3}}$, respectively, we can write the $C P$-conserving relaxation term associated with Fig. 2 as

$$
S^{C P}=\left(\mu_{\nu_{\tau L}}-\mu_{b_{R}^{3}}\right) \Gamma_{M}
$$

with

$$
\begin{aligned}
\Gamma_{M}= & \frac{\left|\tilde{y}_{1} \varphi_{1}(z)+\tilde{y}_{2} \varphi_{2}(z)\right|^{2}}{2 \pi^{2} T} \int_{0}^{\infty} \frac{k^{2} d k}{\omega_{\nu} \omega_{b}} \\
& \times \operatorname{Im}\left[\left(\mathcal{E}_{\nu_{L}} \mathcal{E}_{b}+k^{2}\right)\left(\frac{h_{F}\left(\mathcal{E}_{\nu_{L}}\right)+h_{F}\left(\mathcal{E}_{b}\right)}{\mathcal{E}_{\nu_{L}}+\mathcal{E}_{b}}\right)\right. \\
& \left.-\left(\mathcal{E}_{\nu_{L}} \mathcal{E}_{b}^{*}-k^{2}\right)\left(\frac{h_{F}\left(\mathcal{E}_{\nu_{L}}\right)+h_{F}\left(\mathcal{E}_{b}^{*}\right)}{\mathcal{E}_{b}^{*}-\mathcal{E}_{\nu_{L}}}\right)\right]
\end{aligned}
$$

and

\footnotetext{
${ }^{2}$ In principle, one can have $C P$-violating sources resulting from $C P$ violation in the scalar potential, e.g., see [45]. However, for the purposes of this paper, we only consider the $C P$-violating source listed in Eq. (15).
}

$$
h_{F}(x)=e^{x / T} /\left(1+e^{x / T}\right)^{2} .
$$

With these sources in hand, we now analyze the transport equations (14) in detail. A particle's dynamics are important if it is able to diffuse ahead of the advancing bubble wall. The diffusion time is characterized by a diffusion constant $D_{i}$ (see below) and the bubble wall velocity $v_{w}$ : $\tau_{\text {diff }}=D / v_{w}^{2} \sim 10^{4} / T$ [46] for $v_{w}$ on the order of 0.05 . This time scale is typically shorter than the inverse rate for the EW sphalerons to convert the left-handed number density $n_{L}$ into $B+L, \tau_{\mathrm{EW}} \sim \Gamma_{\mathrm{EW}}^{-1} \sim 10^{5} / T$, where $\Gamma_{\mathrm{EW}} \approx$ $120 \alpha_{W}^{5} T$ and $\alpha_{W}$ is the $\mathrm{SU}(2)_{L}$ fine structure constant[47]. Consequently, we may decouple the equations for $n_{L}$ and $B+L$ generation to a reasonable approximation.

Following Ref. [35], we assume a planar bubble wall profile so that charge densities are functions only of their displacement $z=\left|\vec{x}-\vec{v}_{w} t\right|$ from the bubble wall in its rest frame, where $\vec{x}$ is the coordinate in the plasma rest frame and where the $z<0(z>0)$ region corresponds to the (un) broken phase. We also apply Fick's law to make the replacement $j_{i}^{\mu} \equiv\left(n_{i}, \overrightarrow{j_{i}}\right) \rightarrow\left(n_{i},-D_{i} \vec{\nabla} n_{i}\right)$. Here, $n_{i}$ is the charge density and $D_{i}$ is the diffusion constant which describes how $n_{i}$ is transported away from the bubble wall. Assuming $\mu_{i} / T \ll 1$, the chemical potentials are related to the charge densities as $n_{i}=\left(T^{2} / 6\right) k_{i} \mu_{i}+\mathcal{O}\left(\mu_{i} / T\right)^{3}$ where the $k_{i}$ factor counts the effective degrees of freedom of species $i$ in the plasma. These $k_{i}$ factors are

$$
k_{i}=g_{i} \frac{6}{\pi^{2}} \int_{m_{i} / T}^{\infty} d x x \frac{e^{x}}{\left(e^{x} \pm 1\right)^{2}} \sqrt{x^{2}-m_{i}^{2} / T^{2}}
$$

where $g_{i}$ counts the number of internal degrees of freedom for species $i$ and $m_{i}$ is the effective mass of particle $i$ at temperature $T$. By searching for steady-s tate solutions that only depend on $z$, we can make the replacements $\partial n_{i}(z) / \partial t \rightarrow v_{w} n_{i}^{\prime}(z)$ and $\vec{\nabla}^{2} n_{i}(z) \rightarrow n_{i}^{\prime \prime}(z)$ where the prime denotes differentiation with respect to $z$ and $v_{w} \equiv \partial z / \partial t$ is the velocity of the bubble wall. After these modifications, the Boltzmann equations become a coupled set of second-order differential equations for the charge densities $n_{i}(z)$ with one such equation for each independent particle species coupled to the baryon- and $C P$-violating source in the plasma.

\section{A. Local equilibrium considerations}

The spontaneous breaking of $\mathrm{SU}(3)_{C}$ symmetry implies that one needs to consider the transport dynamics of each color separately. A significant simplification can be made if the dynamics of color and weak isospin singlets can be separated from multiplets. This is what we endeavor to achieve in this section. The only assumption we will require is that we are in a section of parameter space where gauge interactions are fast enough compared to the inverse of the diffusion rate which is controlled by the velocity of the 
advancing bubble wall, $\Gamma_{D} \sim v_{W}^{2} / D$ [48]. For the sake of simplicity we will also assume that scalar interactions involving both colored scalars are also fast enough compared to a diffusion rate to equilibrate the two

$$
\begin{aligned}
n_{Q} & =\sum_{i} n_{t_{L i}}+n_{b_{L i}} \\
n_{T} & =\sum_{i} n_{t_{R i}} \\
n_{B} & =\sum_{i} n_{b_{R i}} \\
n_{U} & =\sum_{i} n_{c_{R i}} \\
n_{L} & =n_{\tau_{L}}+n_{\nu_{\tau L}} \\
n_{H} & =n_{H^{+}}+n_{H^{0}} \\
n_{C} & =\frac{1}{2}\left(\sum_{i \alpha} n_{\chi_{i \alpha}^{2 / 3}}+n_{\chi_{i \alpha}^{-1 / 3}}\right)
\end{aligned}
$$

where the above species are the left-handed third-generation quark doublet; the right-handed top, bottom, and charm; the third-generation left-handed lepton doublet; the Higgs doublet; and the combined colored scalar densities, respectively. Note that $i \in(1,2,3)$ is an $\mathrm{SU}(3)_{C}$ index and $\alpha \in$ $(1,2)$ is an index for the species of leptoquark.

Let us begin with making use of gauge interactions. We will denote the chemical potentials of the two components of an arbitrary $\mathrm{SU}(2)_{L}$ doublet as $\mu_{\uparrow}$ and $\mu_{\downarrow}$, respectively. Also, let us denote the three components of an arbitrary color triplet as $\mu_{i}$ for $i \in 1,2,3$. The remaining $\mathrm{SU}(2)_{C}$ symmetry results in a local equilibrium relation between the first two colors,

$$
\mu_{1}=\mu_{2} .
$$

The result of this is that there are only two independent colors. We can therefore write all components of the $\mathrm{SU}(3)_{C}$ triplet can be written as a linear combination of the color singlet and octet state which we denote as $\mu_{8}$ and $\mu_{S}$, respectively,

$$
\left(\begin{array}{l}
\mu_{S} \\
\mu_{8}
\end{array}\right)=\left(\begin{array}{cc}
2 & 1 \\
\frac{1}{\sqrt{3}} & -\frac{1}{\sqrt{3}}
\end{array}\right)\left(\begin{array}{c}
\mu_{1,2} \\
\mu_{3}
\end{array}\right)
$$

where $\mu_{1,2}$ represents either color since they are in equilibrium.

The assumption of local gauge equilibrium for massive gauge results in the following relations between chemical potentials:

$$
\begin{aligned}
& \mu_{1}-\mu_{3}=\frac{1}{\sqrt{3}} \mu_{8}=\mu_{G_{45}} \\
& \mu_{2}-\mu_{3}=\frac{1}{\sqrt{3}} \mu_{8}=\mu_{G_{67}} \\
& \mu_{\uparrow}-\mu_{\downarrow} \equiv \Delta \mu=-\mu_{W},
\end{aligned}
$$

where $\mu_{\uparrow}\left(\mu_{\downarrow}\right)$ denotes the chemical potential for any weak isodoublet with third component $+1 / 2(-1 / 2)$. The first two lines in Eq. (23) imply

$$
\mu_{G_{45}}=\mu_{G_{67}} \equiv \mu_{G} .
$$

Similarly, any chemical potential of the form $\Delta \mu$ is equal to $-\mu_{W}$. Therefore, the densities of all gauge multiplets in the network of transport equations for color and $\mathrm{SU}(2)_{L}$ singlets can be written in terms of massive gauge boson densities.

Next, we use the $\mathrm{CoB}$ phase conservation laws to eliminate the massive gauge boson densities from all transport equations for color and $\mathrm{SU}(2)_{L}$ singlets. Recall from Sec. III that there are the two charges $Q_{X_{1,2}}$ conserved in the CoB phase. To make use of a conservation law, one must set the sum of the charge asymmetry for all particle species to zero. For example, in the case of the $Q_{X_{1}}$ conservation we have

$$
\sum_{i \in \text { particles }} Q_{X_{1}}\left(\frac{6 n_{i}}{T^{2}}\right)=\sum_{i \in \text { particles }} Q_{X_{1}} \mu_{i} k_{i}=0 .
$$

We find for $Q_{X_{1}}$ the simple relationship

$$
\mu_{G}=-\mu_{W} .
$$

This allows us to eliminate the $\mu_{G}$ in terms of $\mu_{W}$. Next we consider $Q_{X 2}$ conservation. Using Eqs. (23), (24), and (26) we obtain

$\mu_{W}=\frac{3}{16}\left(\frac{1}{6} \mu_{Q_{L}}+\frac{2}{3} \mu_{t_{R}}-\frac{1}{3} \mu_{b_{R}}-\frac{1}{2} \mu_{L}+\mu_{H}+\frac{2}{3} \mu_{C}\right)$,

which can be used to eliminate $\mu_{W}$. We have now achieved our goal of writing $\mu_{8}$ and $\Delta \mu$ in terms of gauge singlet densities.

There exists one additional relationship that allows us to eliminate one more chemical potential. In the CoB phase, the scalar fluctuations about the $\mathrm{CoB}$ vevs are real scalars that can no longer carry any charge, implying vanishing of their chemical potentials,

$$
\mu_{\chi_{\alpha 3}^{-1 / 3}}=0
$$

with $\alpha \in(1,2)$ denoting the leptoquark species. Using Eqs. (22), (23), (24), and (26) we can derive the relation

$$
\mu_{C}=-7 \mu_{W}
$$

Substituting into Eq. (27) and solving for $\mu_{C}$ allows us eliminate the leptoquark chemical potential in favor of the quark, lepton, and Higgs chemical potentials appearing in 
Eq. (27). Thus, the final set of Boltzmann equations need not contain either $\mu_{W}$ or $\mu_{C}$.

To conclude this section we briefly comment on the strong sphaleron rate. Strong sphaleron transitions convert left-handed quarks into right-handed quarks and vice versa. Since we are breaking $\mathrm{SU}(3)_{C}$ through a strongly firstorder phase transition, the sphaleron rate for the third color gets suppressed in the $\mathrm{CoB}$ phase by a factor controlled by the sphaleron energy, $\Gamma_{\mathrm{sph}} \sim \exp \left[-E_{\mathrm{Sph}} / T\right]$, where the sphaleron energy itself is proportional to the color-breaking vev, $v_{\mathrm{cb}}$. Therefore, we can ignore strong sphaleron transitions for the third color. The linear combination of chemical potentials that multiply the remaining $\mathrm{SU}(2)_{C}$ sphaleron rate $\Gamma_{S S}^{(2)}$ in the transport equations is just

$$
\mu_{L 1}+\mu_{L 2}-\mu_{R 1}-\mu_{R 2}
$$

Using Eqs. (22) and (23), one finds that the contributions from the color octets cancel. Recall that we assume that local baryon number is conserved for the first two generations of particles. The result is that Eq. (30) can be written in the form

$$
\mu_{L i}-\mu_{R i}=2\left(8 \mu_{U}+\mu_{T}+\mu_{B}-2 \mu_{Q}\right) .
$$

\section{B. Quantum transport equations}

We now derive the Boltzmann equations for all relevant color and $\mathrm{SU}(2)_{L}$ singlets. To that end, we first construct the Boltzmann equations for the color and isospin components of each field, adding them together to obtain the equations for the color and $\mathrm{SU}(2)_{L}$ singlet densities. To illustrate, consider the right-handed $b$-quark singlet charge density $n_{B}$. Following the steps laid out in the previous subsection we obtain the following equations for the two independent charge densities $n_{b_{R}^{1}}$ and $n_{b_{R}^{3}}$ :

$$
\begin{aligned}
\partial_{\mu} j_{b_{R}^{1}}^{\mu}= & -2 \Gamma_{\chi^{2 / 3}}\left(\mu_{b_{R}^{1}}-\mu_{\chi_{1}^{2 / 3}}-\mu_{\tau_{L}}\right) \\
& -2 \Gamma_{\chi^{-1 / 3}}\left(\mu_{b_{R}^{1}}-\mu_{\chi_{1}^{-1 / 3}}-\mu_{\nu_{\tau L}}\right) \\
& -\Gamma_{S S}^{(2)} \sum_{i=\mathrm{gen}}\left(\mu_{u_{i R}^{1}}+\mu_{d_{i R}^{1}}-\mu_{u_{i L}^{1}}-\mu_{d_{i L}^{1}}\right), \\
\partial_{\mu} j_{b_{R}^{3}}^{\mu}= & -2 \Gamma_{\chi^{2 / 3}}\left(\mu_{b_{R}^{3}}-\mu_{\chi_{3}^{2 / 3}}-\mu_{\tau_{L}}\right) \\
& -2 \Gamma_{\chi^{-1 / 3}}\left(\mu_{b_{R}^{3}}-\mu_{\nu_{\tau L}}\right)-\Gamma_{M}\left(\mu_{b_{R}^{3}}-\mu_{\nu_{\tau L}}\right) \\
& +S^{(\mathrm{CPV}, \mathrm{BLV})}
\end{aligned}
$$

where $\Gamma_{\chi^{2 / 3}}, \Gamma_{\chi^{-1 / 3}}$ are the 3-body rates stemming from the Yukawa interactions in Eq. (4) and $\Gamma_{S S}^{(2)}$ is the strong sphaleron rate associated with nonperturbative $\mathrm{SU}(2)_{C}$ gauge interactions.
Since the gluons associated with $\mathrm{SU}(2)_{C}$ only mediate interactions between the first two components of a $\mathrm{SU}(3)_{C}$ triplet, the strong sphaleron interactions connected with $\mathrm{SU}(2)_{C}$ have no effect on any charge densities corresponding to the third color. Both the 2-body $C P$-conserving rate $\Gamma_{M}$ and the baryon- and $C P$-violating source term $S^{(\mathrm{CPV}, \mathrm{BLV})}$ originate from the interactions with the $\mathrm{CoB}$ vev and thus only appear in Eq. (32b). Moreover, the chemical potential $\mu_{\chi_{3}^{-1 / 3}}$ has vanished due to the formation of CoB vevs. As a consequence, the combination $\mu_{b_{R}^{3}}-\mu_{\nu_{\tau L}}$ is relaxed by both 3 -body, $\Gamma_{\chi^{-1 / 3}}$, and 2-body, $\Gamma_{M}$, interaction rates in Eq. (32b). The factors of two in front of the $\Gamma_{\chi^{2 / 3}}$ and $\Gamma_{\chi^{-1 / 3}}$ rates represent the contributions from both doublets $C_{1}$ and $C_{2}$ whose individual isospin components have been equilibrated by potential operators.

Before taking the singlet combination of Eqs. (32a) and (32b), we simplify them by assuming that all $\chi$ fields have the same mass, implying that all 3-body rates are equal up to the (relatively negligible) difference between the $\tau_{L}$ and $\nu_{\tau L}$ thermal masses, i.e., $\Gamma_{\chi^{2 / 3}}=\Gamma_{\chi^{-1 / 3}} \equiv \Gamma_{C}$. The singlet combination is

$$
\begin{aligned}
\partial_{\mu} j_{B}^{\mu} \equiv & 2 \partial_{\mu} j_{b_{R}^{1}}^{\mu}+\partial_{\mu} j_{b_{R}^{3}}^{\mu} \\
= & -\left(\frac{1}{6}\left(\Gamma_{C}+\Gamma_{M}\right)\left(2 \mu_{B}-\mu_{C}-3 \mu_{L}\right)\right. \\
& \left.+\frac{2}{3} \Gamma_{S S}^{(2)}\left(8 \mu_{U}+\mu_{T}+\mu_{B}-\mu_{Q}\right)\right)+S^{(\mathrm{CPV}, \mathrm{BLV})}
\end{aligned}
$$

where $\mu_{C} \equiv \sum_{\alpha}\left(\mu_{\chi_{\alpha}^{2 / 3}}+\mu_{\chi_{\alpha}^{-1 / 3}}\right)$. Here, we have taken advantage of all equilibrium relations derived in Sec. IVA to write the right-hand side of Eq. (33) entirely in terms of weak isospin and color singlets. Note that to this point we have factored out a factor of 3(2) from the $\mathrm{k}$ factors to account for the components of color triplets (isospin doublets). We now reabsorb these factors into the $\mathrm{k}$ factors appearing in our Boltzmann equations. The final form then becomes

$$
\begin{aligned}
v_{w} B^{\prime}-D_{q} B^{\prime \prime}= & -\left(\Gamma_{C}+\Gamma_{M}\right)\left(\frac{B}{k_{B}}-\frac{C}{k_{C}}-\frac{L}{k_{L}}\right) \\
& +2 \Gamma_{S S}^{(2)}\left(\frac{8 U}{k_{U}}+\frac{T}{k_{T}}+\frac{B}{k_{B}}-\frac{2 Q}{k_{Q}}\right) \\
& +S^{(\mathrm{CPV}, \mathrm{BLV})},
\end{aligned}
$$

where we have expressed the left-hand side of the Boltzmann equation in terms of the singlet density as described in Sec. IV.

Following the steps laid out above for all other independent charge densities in Eq. (20), we obtain

$$
v_{w} U^{\prime}-D_{q} U^{\prime \prime}=-2 \Gamma_{S S}^{(2)} \mathcal{E}_{S S}
$$




$$
\begin{gathered}
v_{w} T^{\prime}-D_{q} T^{\prime \prime}=-2 \Gamma_{S S}^{(2)} \mathcal{E}_{S S}-\Gamma_{H} \mathcal{E}_{H} \\
v_{w} Q^{\prime}-D_{q} Q^{\prime \prime}=4 \Gamma_{S S}^{(2)} \mathcal{E}_{S S}+\Gamma_{H} \mathcal{E}_{H} \\
v_{w} H^{\prime}-D_{L} H^{\prime \prime}=\Gamma_{H} \mathcal{E}_{H} \\
v_{w} B^{\prime}-D_{q} B^{\prime \prime}=-2 \Gamma_{S S}^{(2)} \mathcal{E}_{S S}-\left(\Gamma_{C}+\Gamma_{M}\right) \mathcal{E}_{M} \\
+S^{(\mathrm{CPV}, \mathrm{BLV})} \\
v_{w} L^{\prime}-D_{L} L^{\prime \prime}=\left(\Gamma_{C}+\Gamma_{M}\right) \mathcal{E}_{M}-S^{(\mathrm{CPV}, \mathrm{BLV})} \\
\mathcal{E}_{H} \equiv\left(\frac{T}{k_{H}}-\frac{Q}{k_{Q}}-\frac{H}{k_{H}}\right), \\
\mathcal{E}_{S S} \equiv\left(\frac{8 U}{k_{U}}+\frac{T}{k_{T}}+\frac{B}{k_{B}}-\frac{2 Q}{k_{Q}}\right)
\end{gathered}
$$

where

$$
C=-\frac{7}{10}\left(\frac{1}{6} Q+\frac{2}{3} T-\frac{1}{3} B-\frac{1}{2} L+H\right),
$$

obtained through the combination of Eqs. (29) and (27).

Note that we have assumed the rate for EW sphalerons is much slower than all other rates considered thus far and have, thus, not included the EW sphaleron transition terms in computing the densities. ${ }^{3}$ Consequently, the transport equations should conserve $B+L$. This conservation is manifest for the third-generation fermions, as one can see by adding Eqs. (35b), (35c), (35e), (35b) and noting that the transport equation for the right-handed leptons has a vanishing rhs. For the first- and second-generation fermions, we note that (a) the transport equation for the firstand second-generation down-type right-handed quarks has the same form as Eq. (35a) but with $U \rightarrow D$; (b) the equation for the first- and second-generation left-handed quark doublets has the same form as Eq. (35c) but with vanishing $\Gamma_{H}$; (c) the transport equations for the first- and second-generation left-handed and right-handed leptons also have a vanishing rhs. Consequently, $B+L$ is locally conserved for the first and second generations as well in the limit of vanishing EW sphaleron rate.

In Eqs. (35a), (35b), (35c), (35e), the numerical value of the diffusion constant, $D_{q}$, for all quark states depends on whether $\mathrm{SU}(3)_{C}$ or $\mathrm{SU}(2)_{C}$ is the conserved color symmetry. However, for simplicity, we assume the value $D_{q}=6 / T$ throughout, obtained in $\mathrm{SU}(3)_{C}$ conserving calculations, while for $D_{L}$ we take $100 / T$ [49,50].

\footnotetext{
${ }^{3}$ We will take the resulting left-handed fermion density as input into the EW sphaleron-driven equation for $B+L$ below.
}

The set of transport coefficients excluding the relaxation term which was already given in Eq. (17) are

$$
\begin{gathered}
\Gamma_{H}=\frac{36 y_{t}^{2}}{T^{2}} \mathcal{I}_{F}\left(m_{t_{R}}, m_{Q}, m_{C}\right)+0.13 \alpha_{s} T \\
\Gamma_{C}=\frac{144\left|\tilde{y}_{1}\right|^{2}}{T^{2}} \mathcal{I}_{F}\left(m_{b_{R}}, m_{L}, m_{C}\right)+0.52\left|\tilde{y_{1}}\right|^{2} \alpha_{s} T .
\end{gathered}
$$

The relaxation rates $\Gamma_{H}$ and $\Gamma_{C}$ depend on the function $\mathcal{I}_{F}$ [51] that characterizes the 3-body decays, $t_{R} \rightarrow Q+H$ and $b_{R} \rightarrow L+C$, respectively, and a 4-body scattering contribution proportional to $\alpha_{s}$. Note that, in regions of mass parameter space where $\mathcal{I}_{F}$ due to kinematic blocking, the 4-body term remains nonzero. Also note that for the sake of simplicity we have restricted ourselves to the case where $\tilde{y_{1}}=\tilde{y_{2}}$.

Finally, we consider the nonperturbative $\mathrm{SU}(2)_{C}$ strong sphaleron rate $\Gamma_{S S}^{(2)}$. In Ref. [52], the $N_{C}$ dependence of the strong sphaleron rate was explored. By following their results, we identify the numerical value of the $\mathrm{SU}(2)_{C}$ strong sphaleron rate to be roughly $\Gamma_{S S}^{(2)} \simeq 9 \alpha_{S}^{4} T$.

In the next section, we present our solution of the Boltzmann equations and discuss how this is related to the determination of $Y_{B}$ in CoBBG.

\section{Solving the quantum transport equations and results}

We begin by discussing the parametrization of the set of Boltzmann equations. In principle, the numerical values of all coefficients and source terms in Eq. (35f) are parametrized by 6 unknown model parameters: two tree-level masses $\left[m_{H}(T), m_{C}(T)\right]$ and two complex Yukawa couplings $\left(\tilde{y}_{1}, \tilde{y}_{2}\right)$. However, only the relative phase, $\delta$, is physically relevant. Moreover, for simplicity, we assume that both Yukawa couplings have equal magnitudes $\tilde{y} \equiv\left|\tilde{y}_{1}\right|=\left|\tilde{y}_{2}\right|$. Under these assumptions, the prefactors in Eqs. (15) and (16) become

$$
\begin{aligned}
& \operatorname{Im}\left(\tilde{y}_{1} \tilde{y}_{2}^{*}\right)\left(\varphi_{1} \dot{\varphi_{2}}-\varphi_{2} \dot{\varphi}_{1}\right)=\tilde{y}^{2} \sin \delta \dot{\zeta} \varphi_{C B}^{2} \quad \text { and } \\
& \left|\tilde{y}_{1} \varphi_{1}+\tilde{y}_{2} \varphi_{2}\right|^{2}=\tilde{y}^{2} \varphi_{C B}^{2}(1+\sin (2 \zeta) \cos \delta),
\end{aligned}
$$

respectively, where we remind the reader that $\varphi_{C B}^{2}=\varphi_{1}^{2}+$ $\varphi_{2}^{2}$ and $\tan \zeta=\varphi_{2} / \varphi_{1}$.

The first step in determining $Y_{B}$ in CoBBG is to solve the Boltzmann equations in Eq. (35f). We note that, without a source term for any of the $T, Q$, or $U$ densities, there exists a linear combination of their Boltzmann equations for which the right-hand side vanishes, i.e.,

$$
v_{w}\left(T^{\prime}+Q^{\prime}+U^{\prime}\right)-D_{q}\left(T^{\prime \prime}+Q^{\prime \prime}+U^{\prime \prime}\right)=0 .
$$


Each of these densities diffuses in the plasma at the same rate, which implies that this combination is locally conserved. This means that

$$
T+Q+U=0
$$

thereby eliminating the need for explicit retention of the transport equation for $U=-(Q+T)$. We then employ the novel methods of Ref. [53] to directly solve the reduced set of five Boltzmann equations analytically without recourse to any assumption about the size of the 3-body rates.

For each of the densities, $\mathbb{D}=\{T, Q, H, B, L\}$, we assume the boundary conditions $\mathbb{D}( \pm \infty)=0$. That is, we assume that the electroweak phase transition in which $\mathrm{SU}(3)_{C}$ is restored occurs at a time much larger than the diffusion time scale $\tau_{D}$. For simplicity we also approximate the relaxation rate $\Gamma_{M}$ near the bubble wall as a step function

$$
\Gamma_{M}(z)= \begin{cases}0 & z<0 \text { (unbroken) } \\ \Gamma_{M}\left(10 L_{w}\right) & z>0 \text { (broken) }\end{cases}
$$

where $L_{w}$ is the width of the bubble wall and $10 L_{w}$ is simply a sufficient distance from the wall that $\Gamma_{M}$ has become constant in the $\mathrm{CoB}$ phase. This produces a slight underestimate of the baryon asymmetry, however the error tends to be small [53]. In order to determine the numerical values of $\Gamma_{M}$ and $S^{(\mathrm{CPV}, \mathrm{BLV})}$, we further require the full space-time dependence of the $\mathrm{CoB}$ vev $\varphi_{C B}$ and its angle $\tan \zeta$ across the bubble wall. A detailed calculation of this dependence requires an involved analysis of the full scalar potential, which we defer to future work. For simplicity, we assume a kink profile [54-57]

$$
\begin{aligned}
v_{C B}(z) & =\frac{\xi T}{2 \sqrt{2}}\left(1+\tanh \left(2 \alpha \frac{z}{L_{w}}\right)\right) \\
\zeta(z) & =\frac{\Delta \zeta}{2}\left(1+\tanh \left(2 \alpha \frac{z}{L_{w}}\right)\right)
\end{aligned}
$$

where we take $\alpha=\xi=3 / 2$. Following detailed calculations in the MSSM [58], we assume a conservative value for $\left.\Delta \zeta \equiv \zeta(T)\right|_{z \rightarrow \infty}-\left.\zeta(T)\right|_{z \rightarrow-\infty}$ which we take to be $\Delta \zeta=0.01$. Note that the BAU is directly proportional to $\Delta \zeta$ and the presence of scalar singlets can lift the value of $\Delta \zeta$ by an order of magnitude or more [59].

In Fig. 3 we present the charge densities for the case of a maximal $C P$-violating phase, $\sin \delta=1$, a Yukawa coupling of $\tilde{y}=0.1$, and tree-level masses of $m_{C}(T)=250 \mathrm{GeV}$ and $m_{H}(T)=100 \mathrm{GeV}$. Moreover, to obtain these results we have used the following phase transition parameters $T=250 \mathrm{GeV}, L_{w}=10 / T$, and $v_{w}=0.05$.

We now discuss calculation of the total baryon asymmetry in the $\mathrm{CoB}$ phase. As previously stated, the baryon asymmetry has two components. A space-time varying

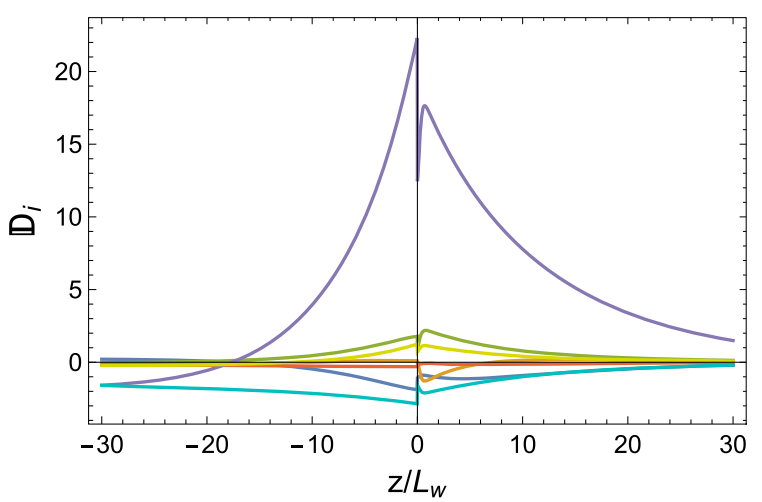

FIG. 3. Charge densities of all relevant species for $m_{C}(T)=$ $800 \mathrm{GeV}, T=250 \mathrm{GeV}, m_{H}(T)=100 \mathrm{GeV}, \sin \delta=1, \tilde{y}=$ $0.1, L_{w}=10 / T$, and $v_{w}=0.05$. Region of positive (negative) $z$ denotes the region of broken (unbroken) $\mathrm{SU}(3)_{C} \times \mathrm{SU}(2)_{L}$.

asymmetry in $B-L$ due to the spontaneous violation of this conserved number within the color-broken phase and the usual component that arises from a total left-handed number density which biases electroweak sphalerons ahead of the advancing bubble wall producing a net $B+L$ asymmetry. Note that deep within the color-broken phase $B+L$ is effectively conserved and this asymmetry will persist into the electroweak phase.

In Figs. 4 and 5 we show how the baryon asymmetry varies as a function of space-time for various values of the leptoquark tree level mass and coupling respectively. The dotted lines in these figures are the contribution to the BAU from the electroweak mechanism, whereas the solid lines are the space-time varying contribution from spontaneous breaking of $B-L$. The total $B-L$ is zero but there is a nonzero density inside the bubble. Note that the coordinate system is the rest frame of the bubble wall; the $B-L$

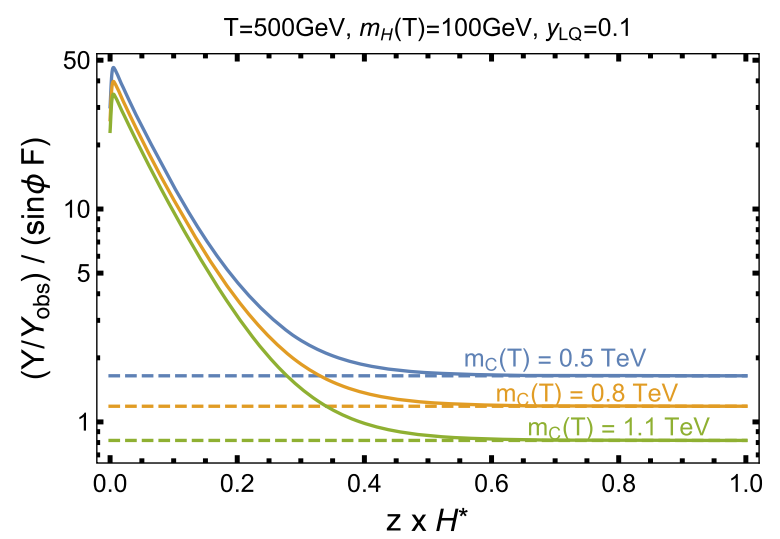

FIG. 4. Variation in BAU due to $m_{C}(T)$ as a function of the space-time variable $z$ normalized by the Hubble length. The BAU has two components: a space-time varying component due to the spontaneous violation of baryon asymmetry and a component due to the EWBG mechanism. The space-time varying component barely penetrates the bubble wall compared to the Hubble length. 


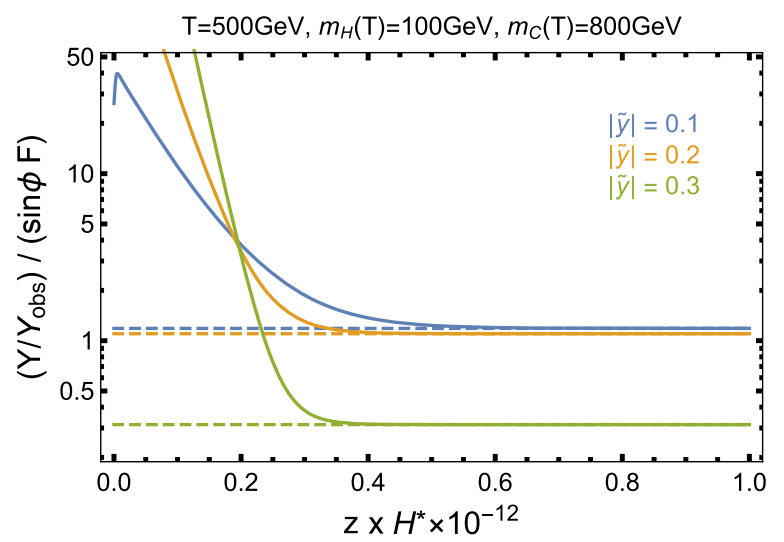

FIG. 5. Variation in BAU due to $y_{L Q}$ as a function of the spacetime variable $z$ normalized by the Hubble length. The BAU has two components: a space-time varying component due to the spontaneous violation of baryon asymmetry and a component due to the EWBG mechanism. The space-time varying component barely penetrates the bubble wall compared to the Hubble length.

density is therefore trapped inside the bubble diluting as the bubble grows. We normalize the space-time variable by the Hubble length at the time of nucleation to highlight that the $B-L$ contribution is very small. Generally, we find that the space-time varying $B-L$ density vanishes at about one trillionth of the Hubble length at the time of nucleation. A significant dilution of this already tiny contribution occurs by the time of recombination. From these figures we see that one can easily produce the BAU for a large range of parameter space during the color-breaking phase transition. The BAU monotonically decreases with $m_{C}(T) / T$ but increases with $\tilde{y}$. The dependence on $m_{C}(T) / T$ is gentle indicating a weak dependence on the leptoquark mass. This is explained by the fact that the leptoquark masses do not enter the functions for the CPV sources; they only appear in the relaxation term $\Gamma_{C}$.

\section{PHENOMENOLOGY}

\section{A. LHC constraints}

At $T=0$, the colored scalars, $C_{1}$ and $C_{2}$, are produced through their strong interactions at the LHC. Under the assumption given in Eq. (6), the scalar decay modes are $\chi^{2 / 3} \rightarrow b_{R} \tau_{L}$ and $\chi^{-1 / 3} \rightarrow b_{R} \nu_{\tau L}$ with unit branching ratios. The CMS Collaboration has recently placed limits on scalar leptoquarks which dominantly decay into these modes by studying their pair production. The dominant pair production mechanisms at the LHC for these colored scalars are through gluon-gluon fusion and quark-antiquark annihilation, for which the cross sections depend only on the scalar mass. At $\sqrt{s}=8 \mathrm{TeV}$, limits have been derived on colored scalars decaying to $b_{R} \tau_{L}$ [60] using an integrated luminosity of $12.9 \mathrm{fb}^{-1}$. A unit branching ratio was assumed and upper limits on the production cross sections were set at the $95 \%$ C.L., yielding the bound of $m_{C_{2 / 3}} \geq 850 \mathrm{GeV}$.
Limits on leptoquarks decaying in the $b_{R} \bar{\nu}_{\tau}$ mode derived by the ATLAS Collaboration are $m_{C_{-1 / 3}} \geq 640 \mathrm{GeV}$ [31].

Aside from direct searches, the colored scalars of CoBBG can also be searched for indirectly by examining their effects on the rates for production and decay of the SM Higgs. At the 1-loop level, their $\mathrm{SU}(3)_{C}$ charges enable them to interfere with top quark loops in gluon-gluon fusion production of the SM Higgs. Also, their $\mathrm{U}(1)_{\mathrm{EM}}$ charges enable them to interfere with both top quark and $W^{ \pm}$loops in Higgs-to-diphoton decay. The modifications of these rates are best expressed as ratios with the SM-valued rates, $R_{\gamma \gamma}\left(R_{g g}\right) \equiv \Gamma_{\gamma \gamma} / \Gamma_{\gamma \gamma}^{\mathrm{SM}}\left(\sigma_{g g} / \sigma_{g g}^{\mathrm{SM}}\right)$. At leading, nontrivial order, one has

$$
\begin{aligned}
R_{\gamma \gamma} & =\frac{\left|F_{1}\left(\tau_{W}\right)+\frac{4}{3} F_{1 / 2}\left(\tau_{t}\right)+N_{c} \sum_{i} Q_{\mathrm{EM}}^{2} \xi_{C_{i}} F_{0}\left(\tau_{C_{i}}\right)\right|^{2}}{\left|F_{1}\left(\tau_{W}\right)+\frac{4}{3} F_{1 / 2}\left(\tau_{t}\right)\right|^{2}} \\
R_{g g} & =\frac{\left|F_{1 / 2}\left(\tau_{t}\right)+\sum_{i} \xi_{C_{i}} F_{0}\left(\tau_{C_{i}}\right)\right|^{2}}{\left|F_{1 / 2}\left(\tau_{t}\right)\right|^{2}},
\end{aligned}
$$

where we sum over the contributions of each colored scalar. Here, we have defined $\tau_{i}=4 m_{i}^{2} / m_{h}^{2}$,

$$
\xi_{C_{i}}=2 \frac{\lambda_{H C_{i}}}{g_{1}} \frac{M_{W}^{2}}{m_{C_{i}}^{2}}
$$

$Q_{\mathrm{EM}}$ is the electric charge of the scalar $C_{i}$, and all loop functions are defined in Ref. [61]. The parameters $\lambda_{H C_{i}}$ are the couplings associated with the Higgs portal operator $H^{\dagger} H C_{i}^{\dagger} C_{i}$. While they do not directly enter the transport computation, they are nevertheless important for the phase transition dynamics.

Using these ratios, we construct the set of signal rates $\mu_{X X}$ associated with Higgs measurements, relative to pure SM-Higgs expectations, i.e.,

$$
\mu_{X X}=\frac{\sigma \cdot \mathrm{BR}}{\sigma^{\mathrm{SM}} \cdot \mathrm{BR}^{\mathrm{SM}}} .
$$

Each signal rate is a function of the Higgs portal couplings $\lambda_{H C_{i}}$ and scalar masses $m_{C_{i}}$ and, for simplicity, we assume that all scalars are degenerate in mass and share the same $\lambda_{H C}$. We then impose constraints on these parameters by performing a global $\chi^{2}$ fit to the current Higgs data ${ }^{4}$ using

$$
\chi^{2}\left(\lambda_{H C}, m_{C}\right)=\sum_{X}\left(\frac{\mu_{X X}^{\mathrm{obs}}-\mu_{X X}}{\Delta \mu_{X X}^{\mathrm{obs}}}\right)^{2},
$$

where $\mu_{i}^{\mathrm{obs}}\left(\Delta \mu_{i}^{\mathrm{obs}}\right)$ are the (uncertainties in the) observed signal rates. The resulting $95 \%$ C.L. limit on the

\footnotetext{
${ }^{4}$ Aside from [62-64], which uses $13 \mathrm{TeV}$ data, the most up-todate signal strengths are taken from 7 and $8 \mathrm{TeV}$ data in Ref. [65]. We use the $13 \mathrm{TeV}$ signal strengths and uncertainties unless the data are unavailable.
} 


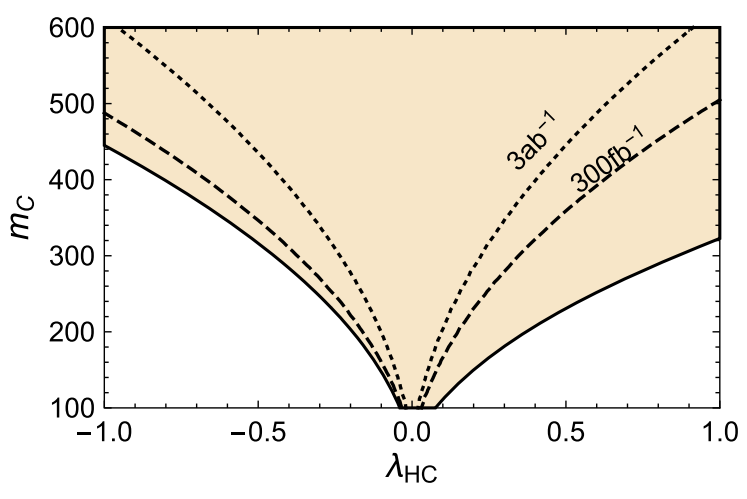

FIG. 6. Shaded region represents the allowed $\left(m_{C}, \lambda_{H C}\right)$ parameter space from current LHC Higgs measurements at the 95\% C.L. The dashed (dotted) lines represent the 95\% C.L. projected sensitivity to this parameter space at the $300 \mathrm{fb}^{-1}$ $\left(3 \mathrm{ab}^{-1}\right)$ high luminosity LHC.

parameters, shown in Fig. 6, implies that, for scalar masses above the current direct search limits $\left(m_{C} \gtrsim 500 \mathrm{GeV}\right)$, a wide range of $\lambda_{H C}$ is open. We also include future projected limits expected from the HL-LHC [66-69], represented by the solid and dashed black contours in Fig. 6.

\section{B. Electric dipole moments}

Searches for permanent electric dipole moments (EDMs) provide constraints on the $C P$-violating phases necessary for producing a baryon asymmetry. (See, e.g., Ref. [11] and references therein. For other recent EDM reviews, see [70-73].) Here, we consider EDM constraints on the $C P$-violating phases present in the leptoquark couplings, $y_{i}$. We find that improvements in experimental sensitivity by many orders of magnitude would be needed to probe the full parameter space of the specific $\mathrm{CoB}$ scenario discussed here.

We work in the effective field theory framework where weak scale particles, $t, W^{ \pm}, Z, H$, and $C_{1,2}$ are considered heavy and integrated out. The effective Lagrangian that results from this is a sum of fermion EDMs, chromo-EDMs (CEDMs), and the three-gluon Weinberg operator [74],

$$
\begin{aligned}
\mathcal{L}_{\mathrm{CPV}}= & -\frac{i}{2} d_{f} \bar{f} \sigma^{\mu \nu} \gamma^{5} f F_{\mu \nu}-\frac{i}{2} \tilde{d}_{q} g_{s} \bar{q}_{i} \sigma^{\mu \nu} \gamma^{5}\left(T^{a}\right)_{i j} q_{j} G_{\mu \nu}^{a} \\
& +g_{s} \frac{C_{W}}{\Lambda^{2}} f^{a b c} G_{\mu \nu}^{a} \tilde{G}^{b \nu \lambda} G_{\lambda}^{c \mu}+\text { H.c. }
\end{aligned}
$$

Here, $F_{\mu \nu}\left(G_{\mu \nu}^{a}\right)$ is the photon (gluon) field strength, $\tilde{G}_{\mu \nu}^{a} \equiv$ $\frac{1}{2} \epsilon_{\mu \nu \alpha \beta} G^{a \alpha \beta}$ is the dual field strength (with $\epsilon_{0123}=+1$ ), and $T^{a}$ and $f^{a b c}$ are the full $\mathrm{SU}(3)_{C}$ generators and structure constants, respectively. Finally, $\Lambda$ is the BSM scale that has been explicitly factored out, whereas the coefficients of the dipole operators retain dimensions of one inverse power of the mass. We will assume that the QCD $\theta$ term, arising at dimension-four in the SM, is removed by the Peccei-Quinn

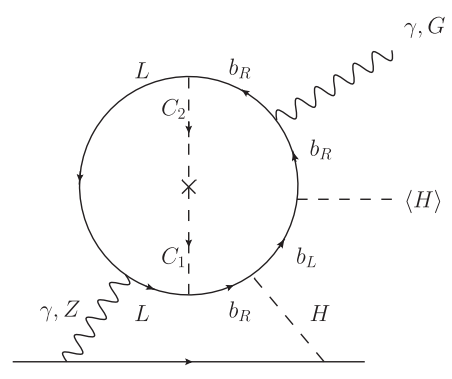

(a)

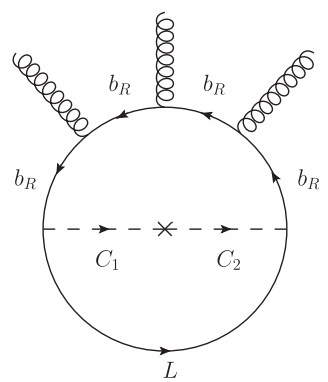

(b)
FIG. 7. Panel (a): Fermion EDMs and chromo-EDMs arise at the 3-loop level, precluding any resulting constructive bounds on the parameter space from these sources. Panel (b): The neutron EDM is sensitive to $C P$ violation in CoBBG through the Weinberg operator. Next-generation experiments searching for neutron EDMs require an improvement of roughly $\mathcal{O}\left(10^{3}\right)$ in order to test the CoBBG scenario.

mechanism [75]. Moreover, we do not consider $C P$-odd four-fermion interactions (generated by tree-level $C_{1,2}$ exchange) as all couplings to first- and second-generation fermions are suppressed.

Elementary fermion EDMs and CEDMs originate first at the 3-loop level from the three loop, Barr-Zee type graphs shown in Fig. 7(a). This loop suppression arises from the need for a different phase in the Yukawa vertices in the fermion loop. Such a diagram requires mixing of $C_{1,2}$ mixing through Higgs portal interactions. ${ }^{5}$ Naive dimensional analysis yields for the electron EDM

$$
\begin{aligned}
d_{e} & \simeq e \frac{\alpha_{\mathrm{EM}}}{4 \pi} \frac{\operatorname{Im}\left(y_{1} y_{2}\right)}{(4 \pi)^{4}} \frac{m_{e} m_{b}^{2}}{m_{C}^{4}} \\
& \sim 5 \times 10^{-36} \operatorname{Im}\left(y_{1} y_{2}\right)\left(\frac{\mathrm{TeV}}{m_{C}}\right)^{4} e \cdot \mathrm{cm} .
\end{aligned}
$$

The scaling with $m_{C}^{-4}$ is not surprising, since the $C_{1}-C_{2}$ mixes via a $\left\langle H^{\dagger} H\right\rangle$ insertion from a Higgs portal in the scalar potential. For $m_{C}=500 \mathrm{GeV}$ this gives $d_{e} \sim 10^{-34} \operatorname{Im}\left(y_{1} y_{2}\right) e \cdot \mathrm{cm}$, far below even the recent ACME bound $\left|d_{e}\right|<8.7 \times 10^{-29} e \cdot \mathrm{cm}$ [76], leaving the $C P$-odd phases unconstrained. Accordingly, we neglect all fermion (chromo-)EDMs.

The neutron EDM receives contributions from the quark EDM and chromo-EDM operators as well as the Weinberg three-gluon operator. For the light quarks, the (chromo-) EDMs will be enhanced compared to $d_{e}$ by $m_{q} / m_{e} \sim 10$, where $m_{q}$ is the light quark mass. The resulting contribution will, nevertheless, be far too small to be experimentally relevant.

\footnotetext{
${ }^{5}$ Note that inclusion of unsuppressed first- and second-generation leptoquark interactions gives rise to one-loop elementary fermion (chromo-)EDMs.
} 
The neutron EDM, $d_{N}$, and the isoscalar $P$ and $T$ odd pion-nucleon coupling $\bar{g}_{\pi}^{0}$ are both sensitive to the Weinberg operator. Following Ref. [71], one can relate both $d_{N}$ and $\bar{g}_{\pi}^{0}$ to the Wilson coefficient of the Weinberg operator,

$$
\begin{aligned}
& d_{N}=\frac{v^{2}}{m_{C}^{2}} \operatorname{Im}\left[C_{W}\right] \beta_{\tilde{G}} \\
& \bar{g}_{\pi}^{0}=\frac{v^{2}}{m_{C}^{2}} \operatorname{Im}\left[C_{W}\right] \gamma_{\tilde{G}}
\end{aligned}
$$

where

$\beta_{\tilde{G}}=[2-40] \times 10^{-7} e \cdot f m, \quad \gamma_{\tilde{G}}=[1-10] \times 10^{-6}$.

Based on the diagram in Fig. 7(b), we estimate $C_{W}$ at the weak scale, obtaining

$$
C_{W}=\frac{g_{s}^{2}}{(4 \pi)^{4}} \operatorname{Im}\left(y_{1} y_{2}\right) f\left(\frac{m_{C}^{2}}{m_{b}^{2}}\right) .
$$

Here, $f$ is a 2-loop function which we identify with that calculated in Ref. [77]. We emphasize that, since the internal scalar lines themselves have $\mathrm{SU}(3)_{C}$ charge and can also emit gluons, the true loop function inevitably differs from that of Ref. [77]. However, we expect such contributions to be suppressed relative to Eqs. (51) and (52) due to their explicit momentum dependence, so we persist for now with the above estimate.

Following Refs. [78-80], the running of the Weinberg operator coefficient from the weak scale to the hadronic scale is given by

$$
C_{W}\left(M_{\mathrm{QCD}}\right)=\left(\frac{\alpha_{s}\left(M_{W}\right)}{\alpha_{s}\left(M_{\mathrm{QCD}}\right)}\right)^{\gamma_{G} /\left(2 \beta_{0}\right)} C_{W}\left(M_{W}\right) .
$$

with anomalous dimension $\gamma_{G}=N_{c}+2 n_{f}+\beta_{0}, \quad \beta_{0}=$ $11-2 / 3 n_{f}$, and $n_{f} \equiv$ the number of active quark flavors. As heavy quark flavors are integrated out at their respective masses, threshold effects arise [81], inducing a shift in $C_{W}$ proportional to the corresponding CEDM. In particular, such a shift occurs at the $b$-quark mass threshold and can lead to significant effects if $\tilde{d}_{b}$ can be generated at the 1-loop level. However, as $C_{1,2}$ couple only to $b_{R}$, generation of $\tilde{d}_{b}$ still arises from the Bar-Zee graphs in Fig. 7(a), rendering the resulting shift completely negligible.

The resulting estimates for the neutron EDM and $\bar{g}_{\pi}^{0}$ are then

$$
\begin{aligned}
d_{N} & \approx[3-60] \times 10^{-25} \frac{v^{2}}{m_{C}^{2}} \operatorname{Im}\left(y_{1} y_{2}\right) f\left(\frac{m_{C}^{2}}{m_{b}^{2}}\right) e \cdot \mathrm{cm} \\
\bar{g}_{\pi}^{0} & \approx[1.5-15] \times 10^{-11} \frac{v^{2}}{m_{C}^{2}} \operatorname{Im}\left(y_{1} y_{2}\right) f\left(\frac{m_{C}^{2}}{m_{b}^{2}}\right)
\end{aligned}
$$

which, for $m_{C}=500 \mathrm{GeV}$, gives $d_{N} \simeq 10^{-28} \operatorname{Im}\left(y_{1} y_{2}\right)$. The current upper limit on the neutron EDM is set at the $90 \%$ C.L. as $\left|d_{n}\right|<[2.9-3.0] \times 10^{-26} e \cdot \mathrm{cm}[82,83]$, implying that next-generation neutron EDM experiments require improvements of $\mathcal{O}\left(10^{2}-10^{3}\right)$ to directly probe the $C P$ violation responsible for baryon production during the $\mathrm{CoB}$ phase transition. The current upper limit on the isoscalar coupling is $\left|\bar{g}_{\pi}^{0}\right|<3.8 \times 10^{-12}$ [73], and we similarly find we are at least two orders of magnitude below this bound for a leptoquark mass of $500 \mathrm{GeV}$. Therefore, contributions to EDMs are indeed constrained within our model. However, we make the following two caveats regarding our analysis:

(1) Under our current assumption of a $C P$-conserving potential, the operators responsible for $C_{1,2}$ mixing do not themselves contribute a phase, and thus precision measurements of EDMs directly constrain the phase responsible for baryon production during the $\mathrm{CoB}$ transition. If this assumption is relaxed, the connection between the $C P$ violation responsible for baryon production and that appearing in EDMs becomes less clear.

(2) The values of $\beta_{\tilde{G}}$ and $\gamma_{\tilde{G}}$ are quite uncertain and span an order of magnitude.

We leave a thorough calculation of each EDM as well as consideration of these issues to a future project.

\section{CONCLUSIONS}

EWBG links the generation of the cosmic baryon asymmetry to electroweak symmetry breaking in the early Universe. In contrast to other theoretically well-motivated scenarios, it is one of the most testable, since it involves new weak scale physics. Not surprisingly, null results for permanent EDMs as well as new particle searches at the LHC tightly constrain EWBG models.

In this work we have relaxed the assumption that today's symmetries have always been symmetries of nature throughout our cosmic history [16-26]. We specifically examine the possibility that $\mathrm{SU}(3)_{C}$ was broken for a period and then subsequently restored [25]. This framework of CoB represents a new EWBG paradigm. We have presented an implementation of this framework that successfully reproduces the BAU without significant finetuning while evading present experimental constraints. The framework is still testable because the leptoquark couplings cannot be arbitrarily small, nor can their masses be arbitrarily heavy.

In $\mathrm{CoB}$, the $\mathrm{BAU}$ is generated during an intermediate color breaking phase transition. We consider the case where color-breaking fields couple to SM fermions so as to avoid stable colored relics. Furthermore, in our implementation the interaction between the color-breaking fields and the standard model fermions conserve $B-L$. As such, the spontaneous breaking and restoration of $\mathrm{SU}(3)_{C}$ is 
associated with spontaneous breaking and restoration of $B-L$. However, during the color-breaking transition a $B+L$ asymmetry is generated through the electroweak mechanism which persists even when $B-L$ is restored. The contribution from the spontaneous violation of $B-L$ is negligible as any such contribution is quickly relaxed away from the bubble wall.

We conclude by noting that our particular implementation of $\mathrm{CoB}$ was a proof of concept. There are other possible implementations of $\mathrm{CoB}$ and to truly test the viability of any particular model one would need to simultaneously examine the phase transition and the transport dynamics. We leave such an examination to future work.

\section{ACKNOWLEDGMENTS}

We thank Lorenzo Sorbo for helpful discussions. G. W. would like to acknowledge that his contribution to this work was partly funded by both the American Australian Association via the Keith Murdoch fellowship as well the Australian Postgraduate Award (APA). M. J. R. M. and P. W. were supported in part under U.S. Department of Energy Contract No. DE-SC0011095.
[1] P. A. R. Ade et al. (Planck Collaboration), Astron. Astrophys. 571, A16 (2014).

[2] A. D. Sakharov, Pis'ma Zh. Eksp. Teor. Fiz. 5, 32 (1967) [JETP Lett. 5, 24 (1967)]; A. D. Sakharov, Usp. Fiz. Nauk 161, 61 (1991) [Sov. Phys. Usp. 34, 392 (1991)].

[3] M. B. Gavela, P. Hernandez, J. Orloff, and O. Pene, Mod. Phys. Lett. A 09, 795 (1994).

[4] P. Huet and E. Sather, Phys. Rev. D 51, 379 (1995).

[5] M. B. Gavela, P. Hernandez, J. Orloff, O. Pene, and C. Quimbay, Nucl. Phys. B430, 382 (1994).

[6] M. Gurtler, E.-M. Ilgenfritz, and A. Schiller, Phys. Rev. D 56, 3888 (1997).

[7] M. Laine and K. Rummukainen, Nucl. Phys. B, Proc. Suppl. 73, 180 (1999).

[8] F. Csikor, Z. Fodor, and J. Heitger, Phys. Rev. Lett. 82, 21 (1999).

[9] Y. Aoki, F. Csikor, Z. Fodor, and A. Ukawa, Phys. Rev. D 60, 013001 (1999).

[10] M. Trodden, Rev. Mod. Phys. 71, 1463 (1999).

[11] D. E. Morrissey and M. J. Ramsey-Musolf, New J. Phys. 14, 125003 (2012).

[12] G. A. White, A Pedagogical Introduction to Electroweak Baryogenesis, IOP Concise Physics (Morgan and Claypool, San Rafael, CA, 2016).

[13] D. Curtin, P. Jaiswal, and P. Meade, J. High Energy Phys. 08 (2012) 005.

[14] A. Katz, M. Perelstein, M. J. Ramsey-Musolf, and P. Winslow, Phys. Rev. D 92, 095019 (2015).

[15] S. Liebler, S. Profumo, and T. Stefaniak, J. High Energy Phys. 04 (2016) 143.

[16] S. Weinberg, Phys. Rev. D 9, 3357 (1974).

[17] R. N. Mohapatra and G. Senjanovic, Phys. Rev. Lett. 42, 1651 (1979).

[18] R. N. Mohapatra and G. Senjanovic, Phys. Rev. D 20, 3390 (1979).

[19] P. Langacker and S.-Y. Pi, Phys. Rev. Lett. 45, 1 (1980).

[20] A. Hammerschmitt, J. Kripfganz, and M. G. Schmidt, Z. Phys. C 64, 105 (1994).
[21] G. R. Dvali, A. Melfo, and G. Senjanovic, Phys. Rev. Lett. 75, 4559 (1995).

[22] G. R. Dvali, A. Melfo, and G. Senjanovic, Phys. Rev. D 54, 7857 (1996).

[23] J. M. Cline, G. D. Moore, and G. Servant, Phys. Rev. D 60, 105035 (1999).

[24] H. H. Patel and M. J. Ramsey-Musolf, Phys. Rev. D 88, 035013 (2013).

[25] H. H. Patel, M. J. Ramsey-Musolf, and M. B. Wise, Phys. Rev. D 88, 015003 (2013).

[26] N. Blinov, J. Kozaczuk, D. E. Morrissey, and C. Tamarit, Phys. Rev. D 92, 035012 (2015).

[27] F. Jona and G. Shirane, Ferroelectric Crystals (Pergamon, New York, 1962).

[28] K. A. Olive et al. (Particle Data Group Collaboration), Chin. J. Phys. C 38, 090001 (2014).

[29] J. M. Arnold, B. Fornal, and M. B. Wise, Phys. Rev. D 88, 035009 (2013).

[30] H. Murayama and T. Yanagida, Mod. Phys. Lett. A 07, 147 (1992).

[31] G. Aad et al. (ATLAS Collaboration), Eur. Phys. J. C 76, 5 (2016).

[32] A. Riotto, Phys. Rev. D 58, 095009 (1998).

[33] M. Carena, M. Quiros, M. Seco, and C. E. M. Wagner, Nucl. Phys. B650, 24 (2003).

[34] T. Konstandin, T. Prokopec, M. G. Schmidt, and M. Seco, Nucl. Phys. B738, 1 (2006).

[35] C. Lee, V. Cirigliano, and M. J. Ramsey-Musolf, Phys. Rev. D 71, 075010 (2005).

[36] J. S. Schwinger, J. Math. Phys. (N.Y.) 2, 407 (1961).

[37] K. T. Mahanthappa, Phys. Rev. 126, 329 (1962).

[38] P. M. Bakshi and K. T. Mahanthappa, J. Math. Phys. (N.Y.) 4, 1 (1963).

[39] P. M. Bakshi and K. T. Mahanthappa, J. Math. Phys. (N.Y.) 4, 12 (1963).

[40] L. V. Keldysh, Zh. Eksp. Teor. Fiz. 47, 1515 (1964) [Sov. Phys. JETP 20, 1018 (1965)].

[41] K.-c. Chou, Z.-b. Su, B.-1. Hao, and L. Yu, Phys. Rep. 118, 1 (1985). 
[42] V. Cirigliano, C. Lee, M. J. Ramsey-Musolf, and S. Tulin, Phys. Rev. D 81, 103503 (2010).

[43] V. Cirigliano, C. Lee, and S. Tulin, Phys. Rev. D 84, 056006 (2011).

[44] T. Liu, M. J. Ramsey-Musolf, and J. Shu, Phys. Rev. Lett. 108, 221301 (2012).

[45] S. Inoue, G. Ovanesyan, and M. J. Ramsey-Musolf, Phys. Rev. D 93, 015013 (2016).

[46] A. G. Cohen, D. B. Kaplan, and A. E. Nelson, Phys. Lett. B 336, 41 (1994).

[47] D. Bodeker, G. D. Moore, and K. Rummukainen, Phys. Rev. D 61, 056003 (2000).

[48] D. J. H. Chung, B. Garbrecht, M. J. Ramsey-Musolf, and S. Tulin, Phys. Rev. Lett. 102, 061301 (2009).

[49] M. Joyce, T. Prokopec, and N. Turok, Phys. Rev. D 53, 2930 (1996).

[50] M. Joyce, T. Prokopec, and N. Turok, Phys. Rev. D 53, 2958 (1996).

[51] V. Cirigliano, M. J. Ramsey-Musolf, S. Tulin, and C. Lee, Phys. Rev. D 73, 115009 (2006).

[52] G. D. Moore and M. Tassler, J. High Energy Phys. 02 (2011) 105.

[53] G. A. White, Phys. Rev. D 93, 043504 (2016).

[54] P. John, Phys. Lett. B 452, 221 (1999).

[55] S. Akula, C. Balazs, and G. A. White, Eur. Phys. J. C 76, 681 (2016).

[56] C. Balazs, G. White, and J. Yue, J. High Energy Phys. 03 (2017) 030.

[57] S. Akula, C. Balazs, L. Dunn, and G. White, J. High Energy Phys. 11 (2017) 051.

[58] J. M. Moreno, M. Quiros, and M. Seco, Nucl. Phys. B526, 489 (1998).

[59] J. Kozaczuk, S. Profumo, L.S. Haskins, and C. L. Wainwright, J. High Energy Phys. 01 (2015) 144.

[60] A. M. Sirunyan et al. (CMS Collaboration), J. High Energy Phys. 07 (2017) 121.

[61] J. F. Gunion, H. E. Haber, G. L. Kane, and S. Dawson, Front. Phys. 80, 1 (2000).

[62] CMS Collaboration, Report No. CMS-PAS-HIG-16-044, 2017.
[63] ATLAS Collaboration, Report No. ATLAS-CONF-2017047, 2017.

[64] M. Aaboud et al. (ATLAS Collaboration), J. High Energy Phys. 12 (2017) 024.

[65] G. Aad et al. (ATLAS, CMS Collaborations), J. High Energy Phys. 08 (2016) 045.

[66] CERN Report No. ATL-PHYS-PUB-2013-014, 2013 (unpublished).

[67] CMS Collaboration, in Community Summer Study 2013: Snowmass on the Mississippi (CSS2013) Minneapolis, MN, USA, 2013, 2013 (unpublished), 1307.7135.

[68] The fifth Annual Large Hadron Collider Physics conference, 2017, Higgs boson measurements at LHC after upgrade.

[69] F. De Almeida Dias, CERN Report No. ATL-PHYS-PROC2015-081, 2015 (unpublished).

[70] M. Pospelov and A. Ritz, Ann. Phys. (Amsterdam) 318, 119 (2005).

[71] J. Engel, M. J. Ramsey-Musolf, and U. van Kolck, Prog. Part. Nucl. Phys. 71, 21 (2013).

[72] T. Chupp and M. Ramsey-Musolf, Phys. Rev. C 91, 035502 (2015).

[73] T. Chupp, P. Fierlinger, M. Ramsey-Musolf, and J. Singh (unpublished).

[74] S. Weinberg, Phys. Rev. Lett. 63, 2333 (1989).

[75] R. D. Peccei and H. R. Quinn, Phys. Rev. Lett. 38, 1440 (1977).

[76] J. Baron et al. (ACME Collaboration), Science 343, 269 (2014).

[77] D. A. Dicus, Phys. Rev. D 41, 999 (1990).

[78] S. M. G. Degrassi, E. Franco, and L. Silvestrini, J. High Energy Phys. 11 (2005) 044.

[79] J. Hisano, K. Tsumura, and M. J. S. Yang, Phys. Lett. B 713, 473 (2012).

[80] W. Dekens and J. de Vries, J. High Energy Phys. 05 (2013) 149.

[81] C. S. L. E. Braaten and T. C. Yuan, Phys. Rev. Lett. 64, 1709 (1990).

[82] C. A. Baker et al., Phys. Rev. Lett. 97, 131801 (2006).

[83] J. M. Pendlebury et al., Phys. Rev. D 92, 092003 (2015). 